\title{
Sur un singulier Métacanthocéphale parasite d'Insectivores (Tenrecinae) de Madagascar et des Comores
}

\author{
Par Robert-Ph. DOLLFUS et Yves-J. GOLVAN
}

C'est à Otto von Linstow (1897) que nous devons la première mention et la première description de l'Acanthocéphale parasite de l'intestin de Tenrec ecaudatus (Schreber 1777) de Lacépède 1799 [= Centetes ecaudatus (S. 1777) Illiger 1811]. Cette description est très succincte et la voici in extenso :

«Länge bis $110 \mathrm{~mm}$, Breite vorn $0,79 \mathrm{~mm}$, hinten 1,66 mm, der Körper ist tänienartig gegliedert. Am Rostellum stehen 20 Reihen von je 12 kleinen Haken; die 9 vorderen Reihen bestehen aus $0,047 \mathrm{~mm}$ langen, die 11 hinteren aus $0,034 \mathrm{~mm}$ grossen Haken letzteren fehlt der Wurzelast. Die merkwürdigen Eier sind $0,107 \mathrm{~mm}$ lang und $0,052 \mathrm{~mm}$ breit; sie sind zweischalig und die äussere Schale ist am hinteren Pole verdickt; von hier gehen unregelmässig gebogene Leisten nach vorn, wo sir zu dicht gegrängten Schlingen zusammentreten; die innere Schale ist $0,081 \mathrm{~mm}$ lang und $0,031 \mathrm{~mm}$ breit. »

La figure 25 du travail de Linstow représente deux crochets, l'un de la région antérieure du rostre muni d'une racine à direction postérieure, l'autre de la région basale et dont la racine est réduite à un petit prolongement supérieur. La figure 26 représente l'œuf avec sa coque externe mince et ridée et sa membrane moyenne épaisse.

Anton Meyer (1932) classe cet Echinorhynchus ovocristatus v. Linstow 1897 au voisinage des Moniliformidae et nous ne pouvons que transcrire ici les quelques lignes qui servent d'introduction à son sous-chapitre « Anhang zur Familie Moniliformidae *.

«Es sind hier zunächst 3 ziemlich gut beschriebene Species anzu führen, die möglicherweise die Aufstellung eines besondern Genus erfordern werden. Alle drei Species sind von Interesse wegen der primitiven Stellung und tiergeographischen Bedeutung ihrer Wirte (Beuteltiere (i.e. Isoodon obesulus et Perameles nasuta), Centetes und Myrmecophaga) und wegen ihrer Verbreitung (Australien, Madagaskar und Süd-Amerika). 》

Ainsi donc Meyer pressentait tout l'intérêt de ces trois espèces de $\mathrm{O}$. von Linstow : Echinorhynchus (s.l.) semoni v. Linstow 1898, Echinorhynchus (s.l.) ovocristatus v. 
Linstow 1897 et Echinorhynchus (s.l.) monechinus v. Linstow 1902, inféodées à des Mammifères primitifs vivant dans ces refuges d'espèces archaïques que sont l'Australie, Madagascar et l'Amérique du Sud.

La première de ces espèces a été réétudiée par Johnston et Edmonds (1952) et classée parmi les Moniliformis sensu stricto. Elle paraît spécifique des Marsupiaux soit strictement carnivores [Phascogale penicillata (Shaw 1800) Temminck 1827], soit des Peramelidae dont le comportement trophique est le même que celui des Insectivores placentaires [Isoodon obesulus (Shaw 1793) Is. Geoffroy St-Hilaire 1817 (= Perameles obesula (S. 1793) Et. Geoffroy St-Hilaire 1803), Isoodon torosus (Ramsay 1878) et Perameles nasuta (Et. Geoffroy St-Hilaire 1804)].

Les deux autres espèces ont été classées éga!ement dans les Moniliformis mais par Petrotschenko (1958) bien qu'il n'ait disposé d'aucun argument nouveau pour justifier une telle assignation.

A notre connaissance, Echinorhynchus (s.l.) monechinus O. v. L. 1902 n'a pas été revu par les Helminthologistes d'Amérique du Sud. Par contre, Echinorhynchus (s.l.) ovocristatus O. v. L. 1897 a été sommairement redécrit sous le nom de Heteracanthorhynchus echinopsi par F. Hörchner en 1962. L'auteur disposait d'un matériel en très mauvais état (deux mâles et trois femelles dont une seule relativement bien conservée) récolté dans l'intestin d'un Tenrecinae de Madagascar, Echinops telfairi Martin 1838.

La présente étude est basée sur un matériel abondant, en parfait état de conservation, et provenant de l'intestin d'un Tenrec ecaudatus (Schreber) tué à Tanykelle (petit îlot situé près de Nossi-Bé au Nord de Madagascar) le 29 octobre 1957 par notre collègue Alain G. Chabaud et d'un autre Tenrec de la même espèce tué par notre collègue Jacques Millot en novembre 1954 à la Grande Comore. Les spécimens sont déposés dans les collections d'Helminthologie du Muséum d'Histoire Naturelle de Paris.

$$
\text { *** }
$$

PROMONILIFORMIS OVOCRISTATUS (O. vON LINSTOW 1897) nov. gen.

(= Echinorhynchus ovocristatus O. von Linstow $1897=$ Moniliformis ovocristatus (O.-v. L. 1897) Petrotschenko $1958=$ Heteracanthorhynchus echinopsi Hörchner 1962.

$$
* *
$$

Le matériel comprend près d'une centaine d'Acanthocéphales adultes des deux sexes tant immatures que parfaitement développés.

La plus grande femelle mesure environ $222 \mathrm{~mm}$ et le plus grand mâle $65 \mathrm{~mm}$. Le corps est pratiquement cylindrique, large de 1,1 à $1,5 \mathrm{~mm}$ chez la femelle et de 0,85 à $1 \mathrm{~mm}$ chez le mâle. Il n'existe pas de pseudo-segmentation comme chez un Moniliformis ou un Mediorhynchus, mais le tronc porte des hachures transversales claires (fig. 1), qui sont formées par les anastomoses transversales du système lacunaire, très rapprochées dans la partie supérieure, beaucoup plus lâches dans la partie postérieure. 
Le proboscis est très petit par rapport à la longueur totale. Il est en massue à grosse extrémité supérieure (fig. $2 a$ et $b$ ) et mesure environ $0,550 \mathrm{~mm}$ de long sur 0,250 à $0,300 \mathrm{~mm}$ de diamètre dans sa partie la plus large. Il est armé de 20 à 24 files longitudinales de 8 à 10 crochets chacune, les 4 ou 5 crochets supérieurs possèdent des racines bien développées et sont de grande taille. Les 4 ou 5 crochets inférieurs sont petits et ont des racines réduites. Le passage des crochets vrais apicaux aux « épines » basales se fait sans transition. La ligne de partage entre crochets et épines présente une certaine obliquité (fig. 2 a) et remonte plus haut sur une des faces latérales que sur l'autre. Les racines des grands crochets ont une extrémité inférieure bulbeuse, leur longueur croît de l'apex vers la base du rostre, encore qu'il existe dans certaines files, et chez certains spécimens seulement, des crochets à racine courte, lesquels précèdent immédiatement la première épine. Les longueurs des racines sont, de haut en bas : $\mathrm{C} 1: 0,049$ à $0,052 \mathrm{~mm} ; \mathrm{C} 2: 0,060 \mathrm{~mm} ; \mathrm{C} 3: 0,068$ à $0,070 \mathrm{~mm} ; \mathrm{C} 4: 0,060 \mathrm{~mm}$; C 5 : 0,052 à 0,048 .

La taille des épines diminue également de haut en bas : E $1: 0,042 \mathrm{~mm}$; E 2 : $0,035 \mathrm{~mm} ; \mathrm{E} 3: 0,021$ à $0,025 \mathrm{~mm} ; \mathrm{E} 4: 0,0175 \mathrm{~mm}$ et E $5: 0,005$ à $0,008 \mathrm{~mm}$. Leurs racines rudimentaires sont bilobées dans leur partie supérieure ce qui leur donne l'aspect d'un cœur de carte à jouer.

Le cou est court, cylindroïde, et porte sur chaque face latérale une grosse papille sensorielle ronde d'environ $0,05 \mathrm{~mm}$ de diamètre. Le réceptacle (fig. 3) mesure de 0,550 à $0,650 \mathrm{~mm}$ de long sur $0,125 \mathrm{~mm}$ de largeur maximum. Il est en forme de massue à grosse extrémité inférieure. Il s'insère à la base du rostre. La structure de sa paroi est très complexe (fig. 4). Comme chez tous les Moniliformidae, l'assise musculaire externe est faite de fibres à direction oblique. Elles sont particulièrement fortes dans la partie inférieure du réceptacle, s'entrecroisant sur les lignes médioventrale et médio-dorsale (fig. 5). Elles deviennent de plus en plus grêles en allant vers la partie supérieure du réceptacle et finissent par ne plus former qu'une toile mince. A l'inverse les fibres de l'assise circulaire interne sont faibles dans la partie inférieure du réceptacle et deviennent de plus en plus puissantes vers la partie supérieure, formant une succession d'anneaux épais. Chacun de ces anneaux est formé d'une sorte de raphé équatorial sur lequel s'insèrent et divergent à la manière des nervures secondaires d'une feuille les fibrilles musculaires.

Ce réceptacle présente 2 orifices d'où sortent les muscles rétracteurs et le ligament antéro-postérieur du tronc. L'un de ces orifices sub-termino-ventral (comme chez les autres Metacanthocephala à l'exclusion des Moniliformidae), l'autre est terminal (fig. 5). Les lemnisques sont beaucoup plus longs que le réceptacle (fig. 1-3) filamenteux, creusés d'un canal central

FIG. 1. - Mâle adulte de Promoniliformis ovocristatus (O.v.L. 1897) de $27 \mathrm{~mm}$ de long (Gde Comore)

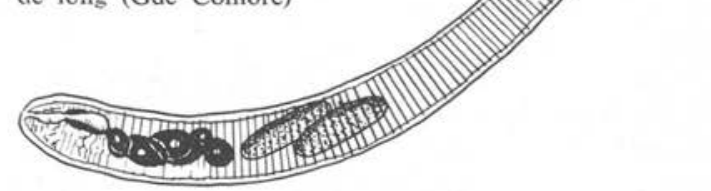



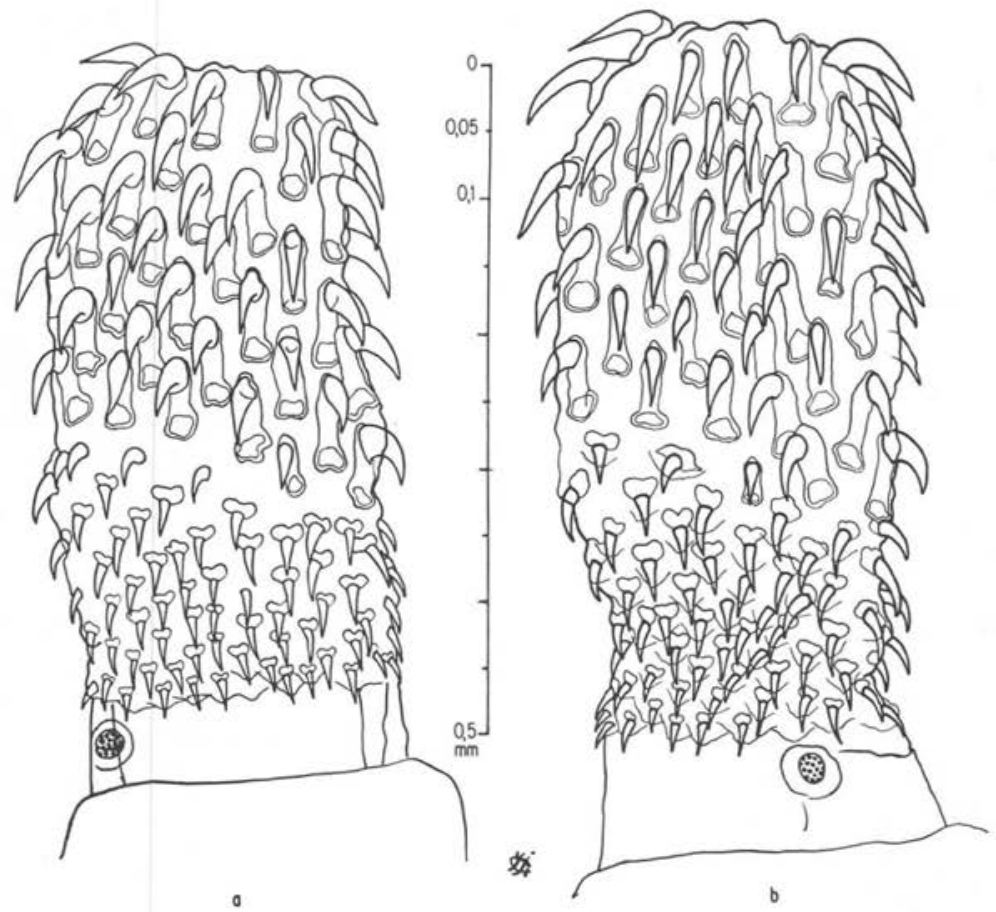

Fig. 2. - Proboscis d'un mâle (a) et d'une femelle $(b)$ adultes de $P$. ovocristatus (O.v.L. 1897) le premier en vue ventrale, le second en vue latérale ce qui montre l'obliquité de ligne d'insertion de la première couronne d'épines. Remarquer également les petits crochets à racines réduites

(fig. 6), contenant généralement l'un 4 et l'autre 5 noyaux géants sphéroïdaux placés immédiatement en-dessous du fond du réceptacle.

Le ganglion cérébroïde est fusiforme, placé contre la face ventrale du réceptacle, un peu au-dessus de son fond.

La paroi du tronc est épaisse. Le système lacunaire comporte deux canaux longitudinaux principaux l'un ventral et l'autre dorsal, reliés par un système complexe d'anastomoses. Dans la partie postérieure (fig. 21), ce réseau a un aspect réticulaire à mailles grossièrement polyédriques. Dans la partie moyenne du tronc (fig. 7) chaque anastomose se divise en 2 branches de calibre égal qui divergent en $\mathrm{V}$ pour se jeter dans le canal principal. Dans la partie antérieure cette disposition tend à perdre de sa netteté l'anastomose s'abouche souvent directement dans le tronc principal.

L'appareil génital mâle qui, chez les spécimens juvéniles, occupe une portion non négligeable de la longueur du tronc, ne semble pas s'accroître beaucoup au cours de la maturation. geable de la longueur du tronc, ne semble pas s'accroître beaucoup au cours de la maturation sexuelle. Il en résulte que, chez les mâles parfaitement mûrs, il n'occupe plus qu'une fraction 


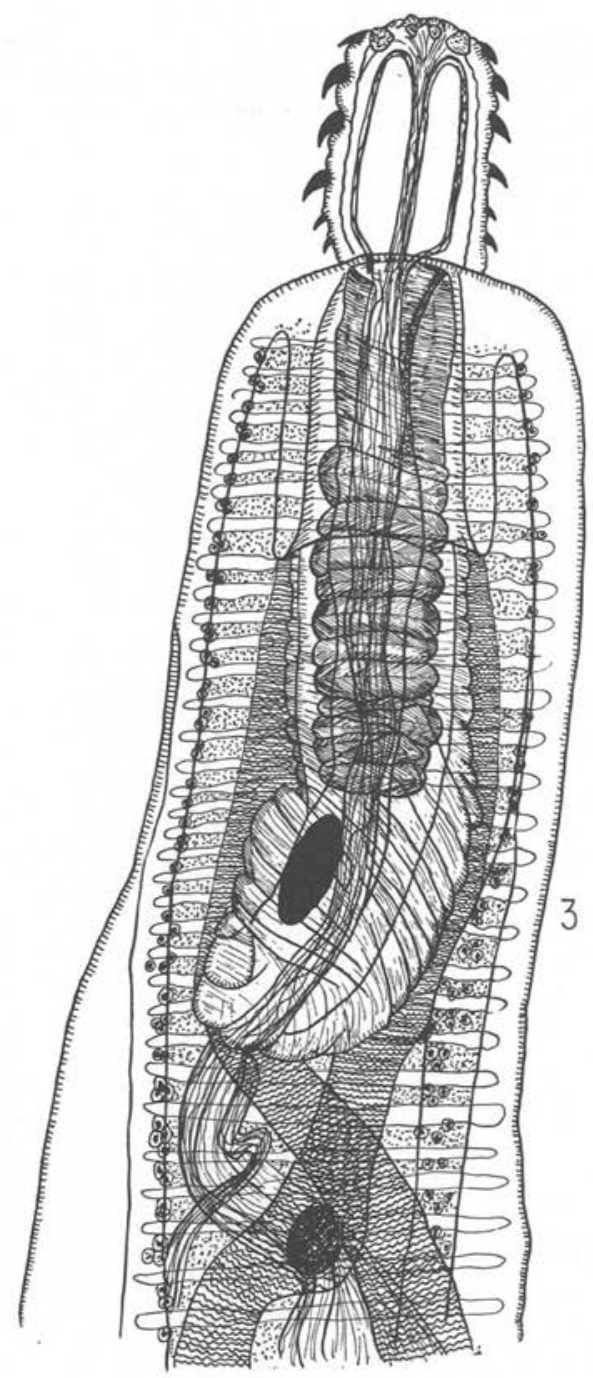

Fig. 3. - Praesoma d'une femelle de P. ovocristatus (O.v.L. 1897)

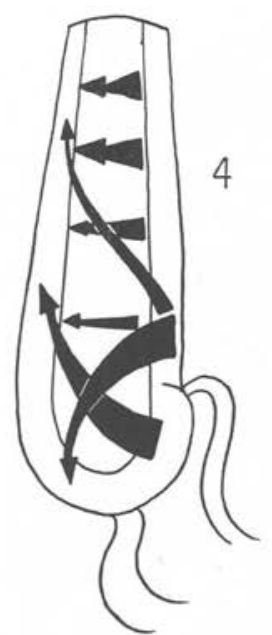

Fig. 4. - Schéma des fibres musculaires formant la double paroi du réceptacle de $P$. ovocristatus (O.v.L. 1897)

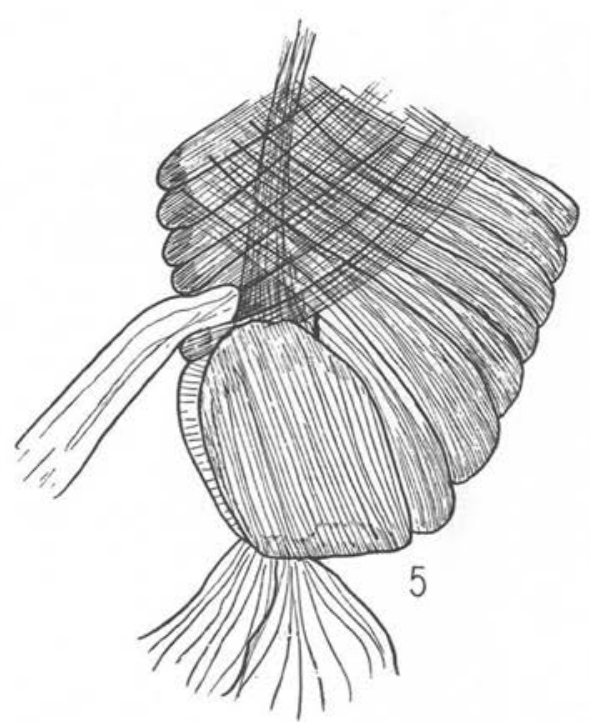

Fig. 5. - Vue latéro-ventrale de l'extrémité inférieure du réceptacle de $P$. ovocristatus (O.v.L. 1897) 


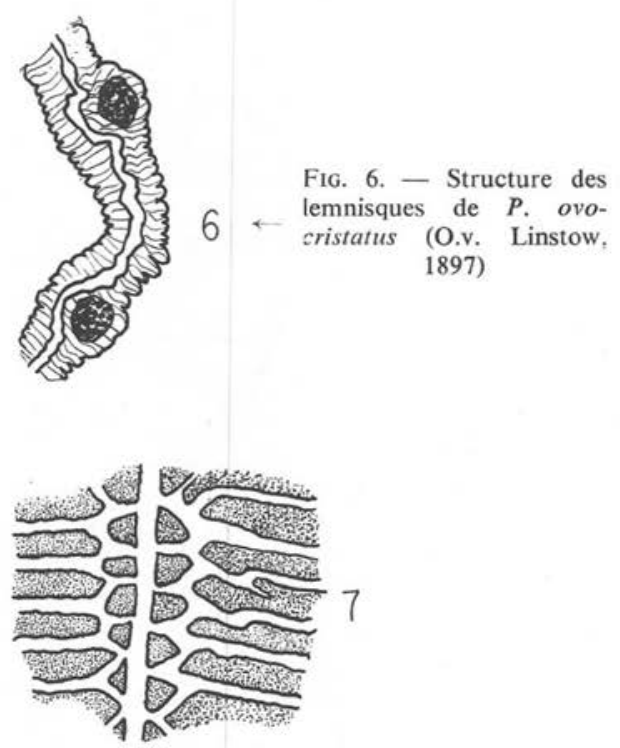

Fig. 7. - Canaux lacunaires et système des anastomoses dans la partie moyenne du tronc de

P. ovocristatus (O.v.L. 1897)

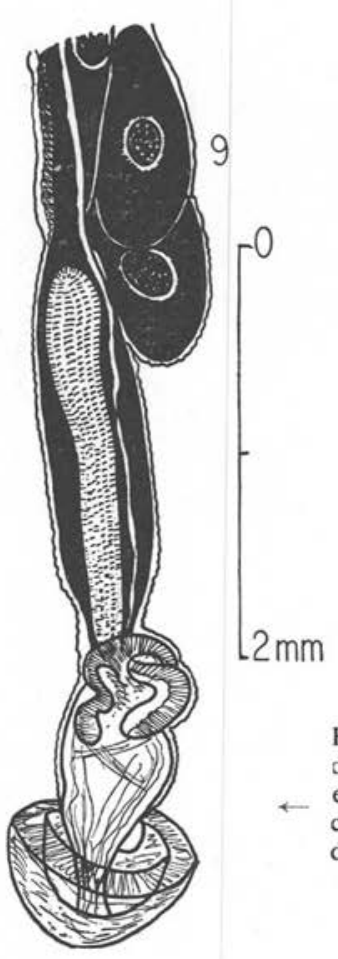

FIG. 8. - Appareil génital mâle de $P$. ovocristatus (O.v.L. 1897)

Fig. 9. - Appareil génital mâle de P. ovocristatus (O.v.L. 1897) en vue latérale montrant les canaux cémentaires cheminant de part et d'autre de l'organe de Säfftigen

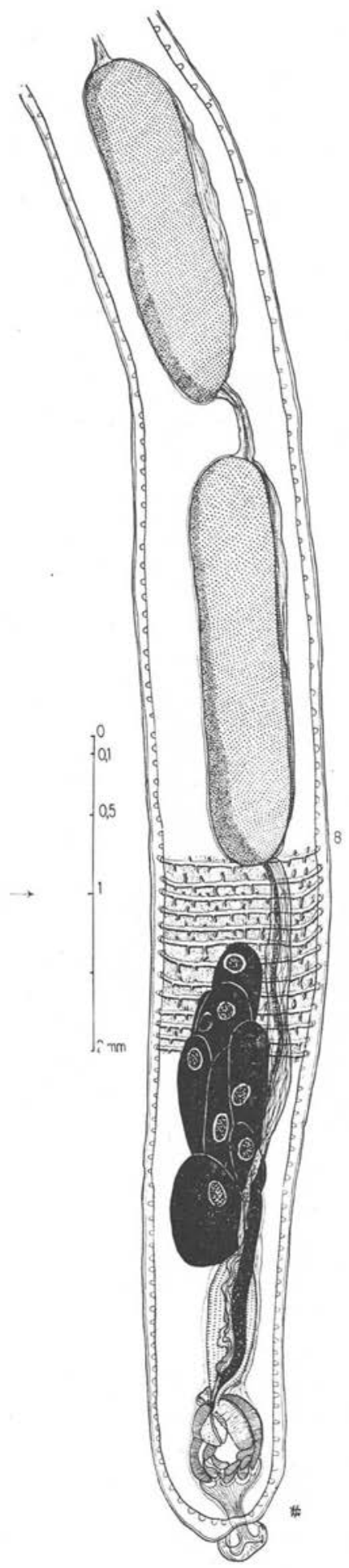




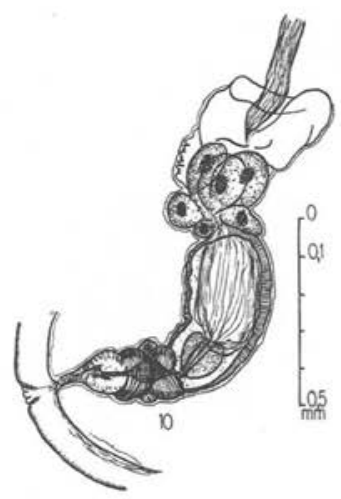

Fıg. 10. - Appareil génital femelle de $P$. ovocristatus (O.v.L. 1897)

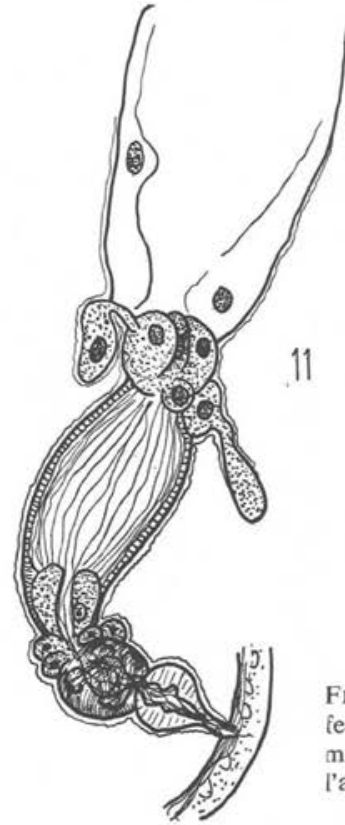

F1G. 11. - Appareil génital femelle d'un autre spécimen montrant les diverticules de l'appareil sélecteur des embryophores

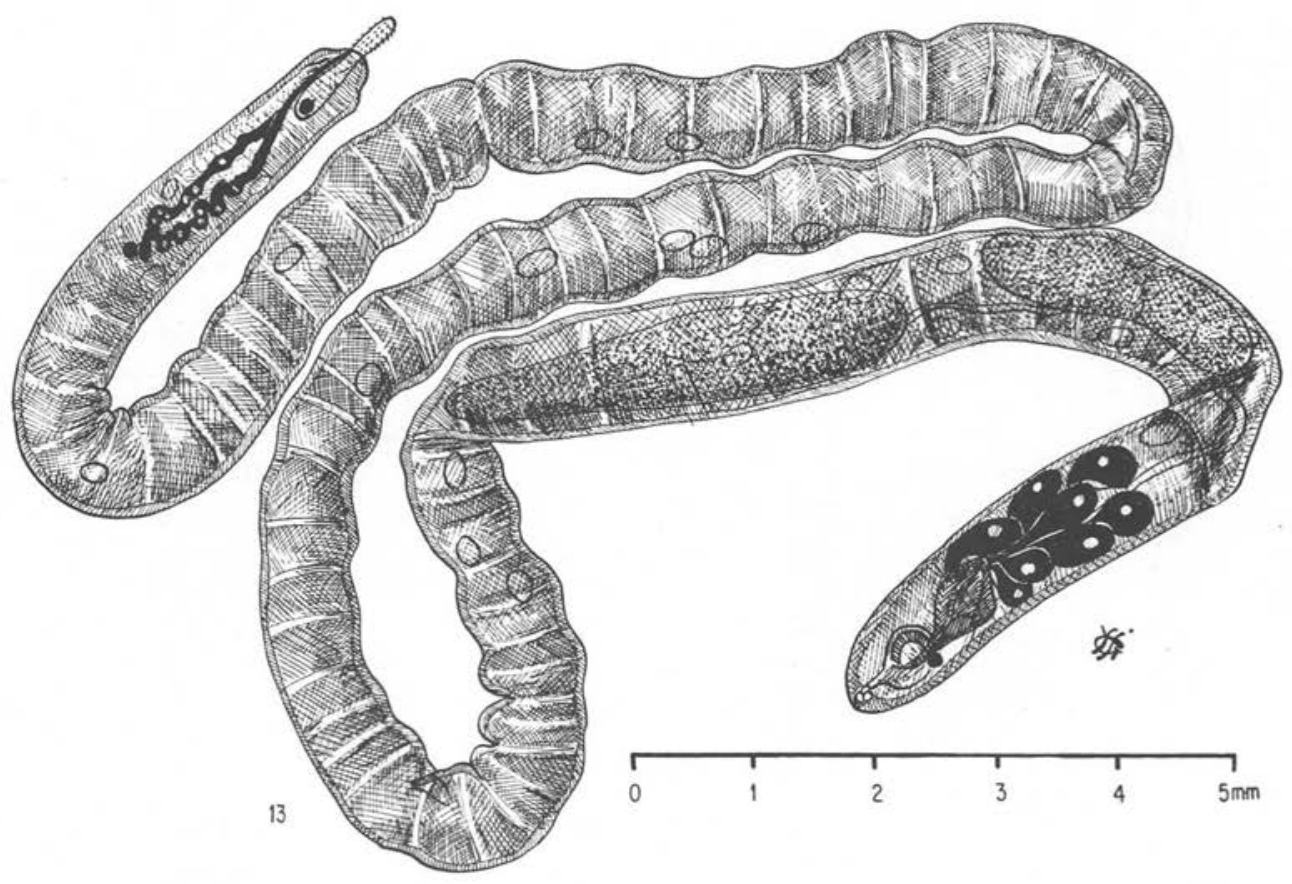

FIg. 13. - Moniliformis moniliformis mâle adulte des Mérions d'Iran 

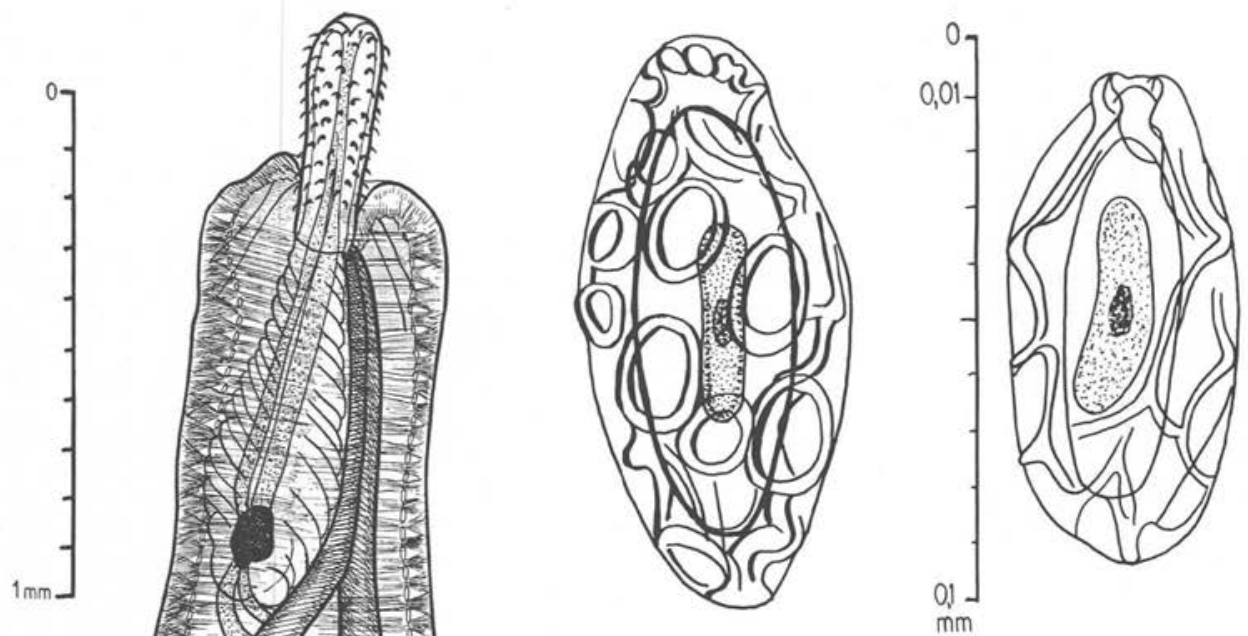

Fig. 12. - Embryophores de P. ovocristatus (O.v.L. 1897)

minime et toute postérieure de la cavité générale (1/6 environ). Testicules ellipsoïdaux (fig. 8 et 9) allongés, de 2,2 à $3 \mathrm{~mm}$ de long sur $0,5 \mathrm{~mm}$ de diamètre, placés l'un derrière l'autre faiblement séparés ou en contact. 8 glandes sphérö̈dales, disposées très irrégulièrement en 4 paires, contenant chacune un seul noyau géant sphéroïdal. Leurs canaux excréteurs cheminent de part et d'autre de l'organe de Säfftigen (fig. 9). Organe de Säfftigen en massue à grosse extrémité supérieure d'environ $1 \mathrm{~mm}$ de long. Bourse copulatrice centrée par un pénis en cone court, avec chape musculaire épaisse, présentant des digitations courtes et obtuses en petit nombre (6 ou 8). Orifice génital terminal. Appareil génital femelle très court (fig. 10 et 11) n'ayant guère qu'1 $\mathrm{mm}$ de longueur totale. Cloche utérine à paroi très mince, se continuant insensiblement avec le sac ligamentaire, contenant 2 noyaux géants. Appareil sélecteur comprenant 6 grosses cellules dont les 2 latérales forment 2 diverticules conte-

Fig. 14. - Praesoma de M. moniliformis des Mérions d'Iran 

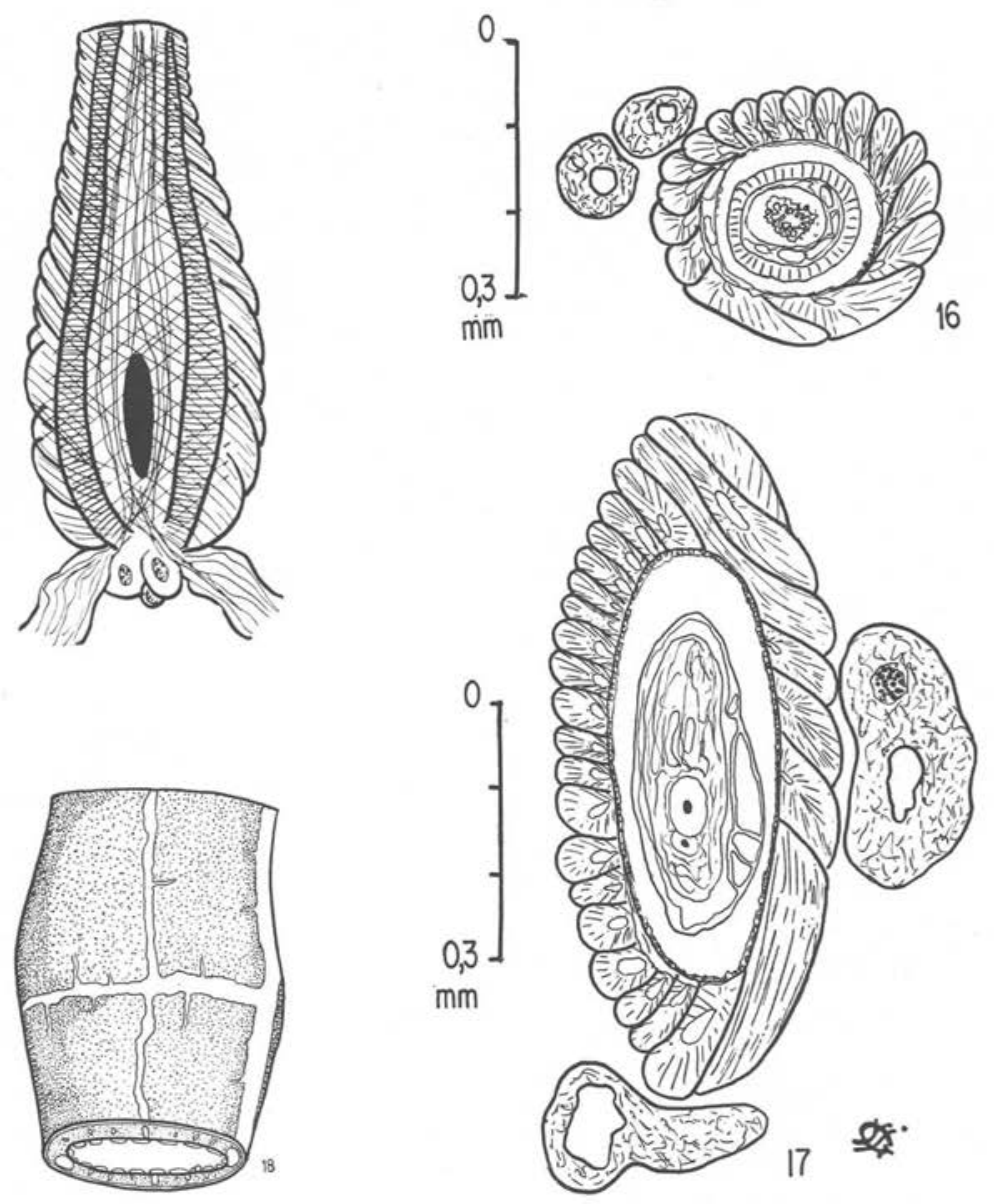

FIG. 15. - Réceptacle de $M$. moniliformis

FIG. 16. - Coupe histologique du réceptacle de $M$. moniliformis dans sa partie supérieure

FIg. 17. - Coupe histologique du réceptacle de $M$. moniliformis dans sa partie inférieure

Fig. 18. - Vue latérale d'un «anneau» de la partie moyenne du tronc d'un $M$. moniliformis mon-

nant souvent des embryophores. Portion tubulaire de l'utérus courte (environ $0,3 \mathrm{~mm}$ ), mais large (environ $0,2 \mathrm{~mm}$ ) à paroi musculaire épaisse. Vagin possédant 2 sphincters subégaux. Atrium piriforme. Vulve terminale. Embryophores très caractéristiques, d'environ $0,105 \mathrm{~mm}$ de long sur $0,041 \mathrm{~mm}$ de diamètre (fig. 12), lorsqu'ils sont parfaitement développés. La membrane externe est mince, papyracée, ridée très irrégulièrement (l'aspect varie considérablement d'un embryophore à l'autre). La membrane moyenne est au contraire épaisse. Acanthor n'ayant guère que $0,03 \mathrm{~mm}$ de long (mais il est possible qu'à frais sa taille soit bien plus grande). 

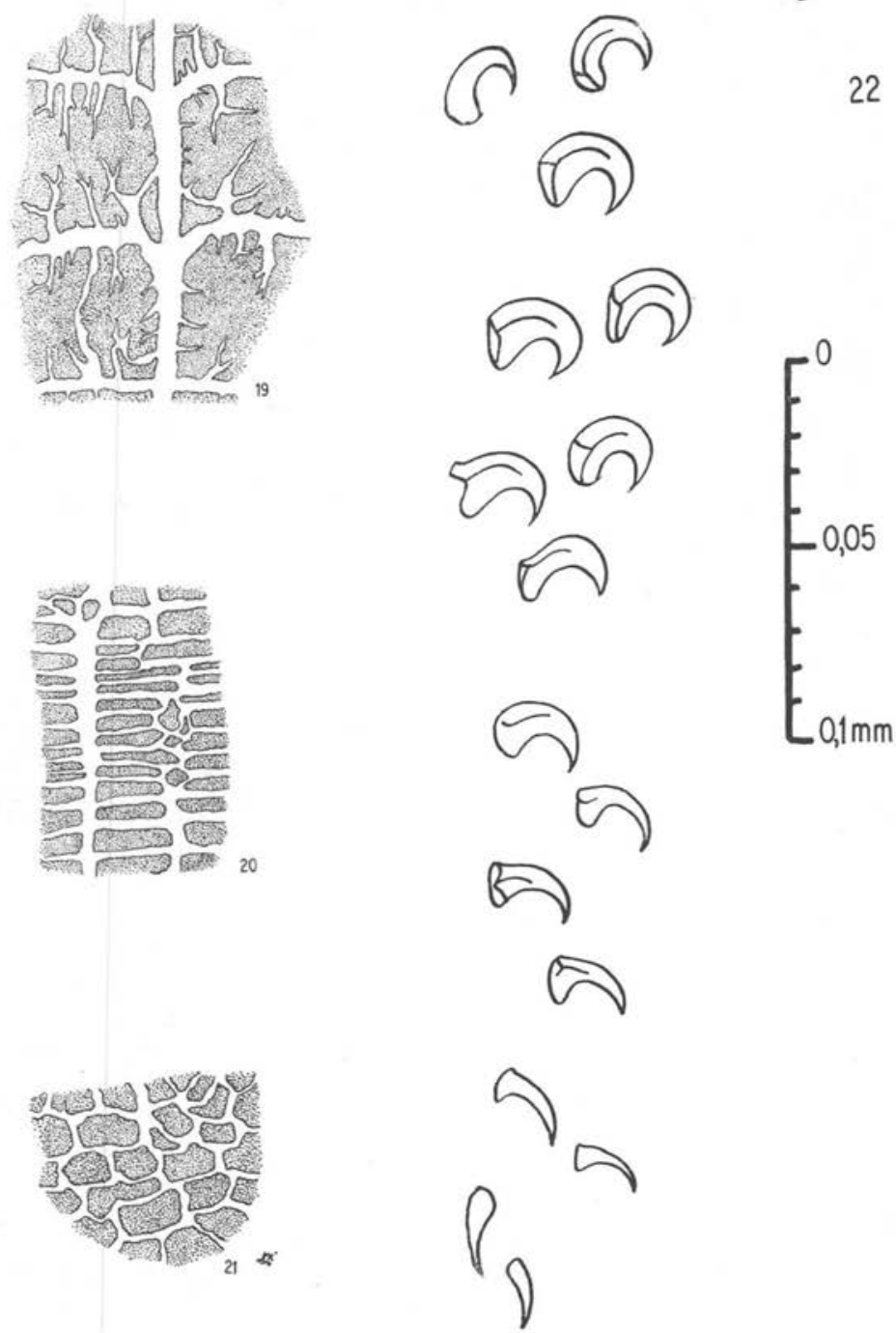

FIG. 19. - Réseau lacunaire de la partie moyenne du tronc de $M$. moniliformis. Comparer cette figure avec la figure 7 et remarquer l'atrophie de la branche supérieure des anastomoses transversales

Fig. 20. - Réseau lacunaire dans la partie antérieure du tronc de $M$. moniliformis. Remarquer que l'abouchement des anastomoses dans le tronc principal se fait sans division préalable

FIG. 21. - Réseau lacunaire dans la partie postérieure du tronc de P. ovocristatus (O.v.L. 1897) montrant sa structure réticulaire irrégulière

F1G. 22. - Crochets du rostre d'un $M$. moniliformis des Mérions d'Iran montrant leur forte courbure, le passage progressif et non brutal des crochets vrais apicaux aux épines basales et le faible développement des racines 


\section{Discussion :}

Il rie nous a pas paru possible de considérer cette espèce comme un vrai Moniliformis car nombre de détails ne correspondent pas à la diagnose de ce genre créé par Travassos en 1915. Nous avons été amenés à comparer notre espèce du Tenrec à un Moniliformis authentique: $M$. moniliformis siciliensis Petrotschenko 1958 dont l'un de nous a récolté plusieurs centaines d'exemplaires chez les Mérions du Kurdistan iranien.

Les Moniliformis montrent une pseudo-segmentation extrêmement nette (fig. 13) mais qui n'apparaît que chez les adultes de grande taille alors que les jeunes adultes ont un habitus qui rappelle celui de l'espèce malgache. Cette pseudo-segmentation indique donc déjà un degré d'évolution plus grand chez les Moniliformis. En second lieu, chez les Moniliformis, l'assise musculaire oblique externe du réceptacle a la même importance du haut en bas de l'organe. C'est ce qui apparaît sur les figures 14 et 15 et également sur les schémas 16 et 17 exécutés d'après des coupes histologiques. Mais l'assise interne est toujours mince alors que chez les autres Metacanthocephala plus primitifs cette circulaire interne présente un très fort développement, en particulier sur la face dorsale. De plus, la présence d'une fente subtermino-ventrale par où sort un faisceau rétracteur permet de rapprocher notre espèce des autres Métacanthocéphales, alors que chez Moniliformis les orifices du réceptacle sont terminaux comme chez les Palaeacanthocephala.

Le système lacunaire est également plus évolué chez les vrais Moniliformis que chez notre Acanthocéphale du

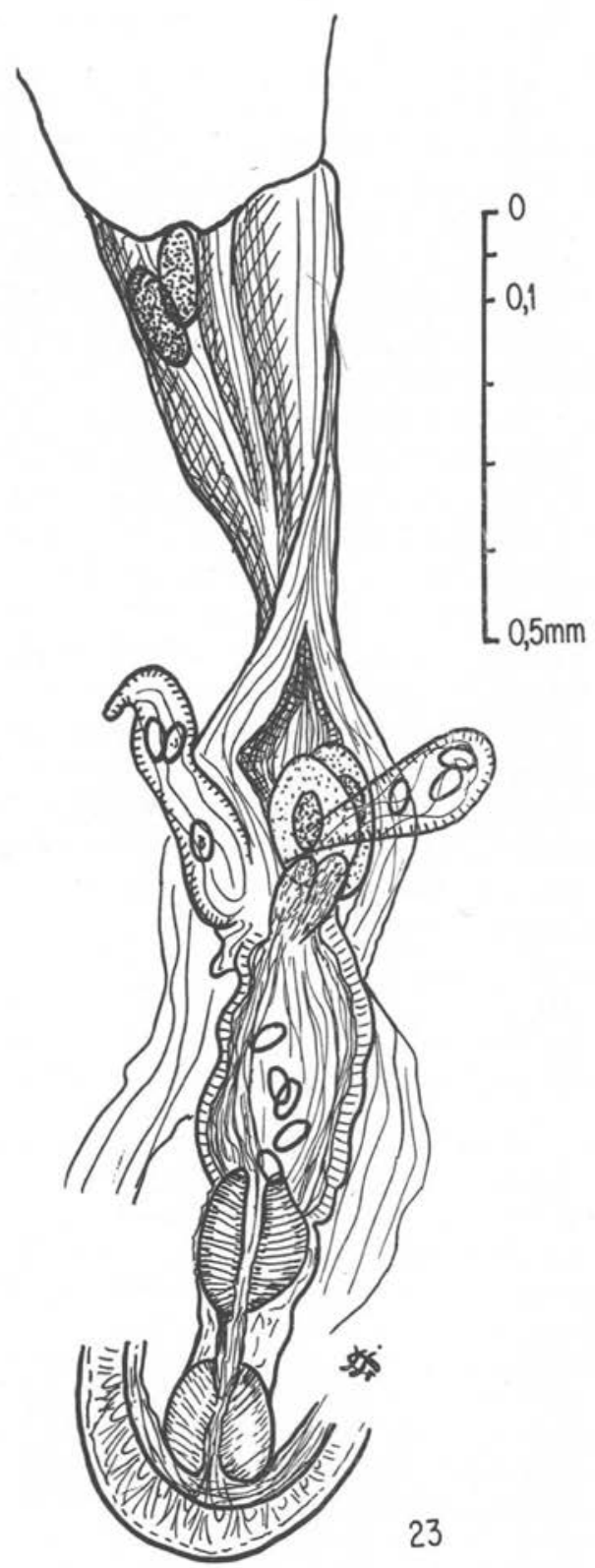

Fig. 23. - Appareil génital femelle de $M$. moniliformis des Mérions d'Iran 
Tenrec. On sait que l'étude des noyaux géants sous-cuticulaires et des modalités de leur fragmentation permet de conclure que les modifications à la fois ontogéniques et phylogéniques se font de l'avant vers l'arrière et que l'extrémité postérieure du tronc conserve longtemps des caractères archaïques alors qu'ils ont déjà disparu de la partie antérieure (ce phénomène n'est d'ailleurs nullement l'apanage des Acanthocéphales et se retrouve dans d'autres groupes). Chez les Moniliformis le système lacunaire forme dans la partie postérieure du tronc, un réseau dont les mailles tendent à prendre déjà la disposition transversale. Dans la partie moyenne du tronc chaque « anneau » (fig. 18) est parcouru, dans sa partie la plus large, par une anastomose transversale. Vers la partie moyenne des faces latérales cette anastomose émet perpendiculairement et au même niveau, deux gros rameaux, l'un ascendant, l'autre descendant qui vont confluer avec leurs homologues nés des anastomoses transversales des anneaux voisins. Il s'ensuit que ces faces latérales sont parcourues chacune par un vaisseau longitudinal, bien plus petit que les deux vaisseaux principaux dorsal et ventral, mais cependant très visible à frais sous forme d'une ligne blanche. On peut donc dire que chez les Moniliformis, en plus des canaux principaux dorsal et ventral à disposition primitive, s'esquisse l'apparition de canaux secondaires latéraux tels qu'on les rencontre chez les plus évolués des Acanthocéphales.

Si l'on étudie maintenant l'abouchement de l'anastomose transversale dans le canal principal dorsal ou ventral on voit que cette anastomose se divise bien en deux branches divergentes comme dans l'espèce malgache mais que l'une de ces branches, l'antérieure, est beaucoup plus grê:e que la postérieure (fig. 19). Lorsque l'on suit la succession de ces anastomoses en allant vers l'extrémité antérieure du tronc, on voit à la fin, disparaître cette branche antérieure (fig. 20) et l'anastomose transversale se jeter directement, sans se diviser, dans le tronc principal.

Pour toutes ces raisons, nous proposons la création du genre Promoniliformis nov. gen. dont la diagnose peut s'énoncer comme suit :

Moniliformidae parasites à l'état adulte de l'intestin des Tenrecinae de toute la sous-région malgache. Corps de grande taille, régulièrement cylindrique, sans succession de dilatations moniliformes. Proboscis relativement petit, en massue à grosse extrémité supérieure, armé de files longitudinales de crochets de 2 types: des crochets vrais, apicaux, à racines bien développées, de forme simple, des «épines» basales, à racines rudimentaires cordiformes. Cou court, portant une paire de papilles sensorielles latérales. Réceptacle inséré à la base du rostre possédant une double paroi, avec une assise oblique externe sourtout puissante à l'extrémité inférieure du réceptacle et une assise circulaire interne surtout puissante à l'extrémité supérieure, avec une fente sub-termino-ventrale, ganglion cérébroïde fusiforme, placé contre la paroi ventrale un peu au-dessus du fond du réceptacle. Lemnisques très longs avec un gros canal axial et contenant dans leur partie moyenne, un petit nombre de noyaux géants sphéroïdaux. Canaux principaux du système lacunaire dorsal et ventral, unis par des anastomoses transversales se divisant en 2 branches divergeant en $\mathrm{V}$ avant de se jeter dans le canal principal. Organes génitaux mâles n'occupant que le 1/6 postérieur du tronc. 8 glandes cémentaires sphéroïdales contenant chacune un noyau géant sphéroïdal. Appareil génital femelle très court. Cloche utérine à paroi très mince, se continuant sans limite nette avec les sacs ligamentaires. 
Vagin à double sphincter. Orifices génitaux terminaux dans les 2 sexes. Embryophores mûrs à coque externe mince, papyracée, à coque moyenne épaisse.

ESPÈCE TYPE : Promoniliformis ovocristatus (O. von Linstow 1897) parasite de Tenrec ecaudatus (Schreber) et de Echinops telfairi Martin à Madagascar et aux Comores.

La diagnose de la famille des Moniliformis Van Cleave 1924 doit être modifiée comme suit :

Gigantorhynchida parasites, à l'état adulte, du tube digestif des Mammifères dont les hôtes intermédiaires sont des Arthropodes et probablement des Insectes terrestres. Corps de taille moyenne à grande, présentant souvent une pseudo-segmentation au niveau du tronc qui n'intéresse que la paroi. Proboscis de petite taille, cylindroïde ou en massue, portant des crochets assez nombreux disposés selon des files longitudinales. Cou court avec papilles sensorielles sur les faces latérales. Réceptacle présentant une assise musculaire oblique externe et une circulaire interne. Ganglion cérébroïde placé dans la partie inférieure du réceptacle. Lemnisques longs, filamenteux, contenant quelques noyaux géants sphéroïdaux. Organes génitaux mâles confinés dans la partie postérieure du tronc, huit glandes cémentaires sphéroïdales disposées plus ou moins régulièrement en quatre paires et contenant chacune un noyau géant sphéroïdal. Appareil génital femelle très court avec cloche utérine à paroi très mince. Orifices génitaux terminaux dans les deux sexes. Pas de protonéphridies. Embryophores ellipsoïdaux à coque externe mince.

La famille comprend deux genres : Moniliformis Travassos 1915 (= Echinorhynchus Zoega in O. F. Müller 1776 p. p. = Gigantorhynchus Hamann 1892 p. p. $=$ Hormorhynchus Ward 1917) et Promoniliformis n. gen.

$$
* *
$$

\section{Résumé :}

Grâce à un matériel abondant et en parfait état de conservation provenant de Madagascar et des Comores nous avons pu redécrire l'Echinorhynchus ovocristatus décrit en 1897 par Otto von Linstow parasite de l'intestin des Tenrecinae de la sous-région malgache. Les caractères de cette espèce qui sont les uns hyperévolués, les autres archaïques nous ont amenés à proposer la création, dans la famille des Moniliformidae Van Cleave 1924, d'un second genre : Promoniliformis nov. gen, dont cette espèce sera le type. Nous l'avons comparée aux Moniliformis sensu stricto. La création de ce nouveau genre et l'étude de ses particularités nous obligent à modifier quelque peu la diagnose de la famille des Moniliformidae. 


\section{Bibliographie}

Haman (O.), 1892. - Das System der Acanthocephalen. Zoolog. Anzeig., XV, n 392, 195-197.

HöRCHNER (F.), 1962. - Zur Helminthenfauna des Igeltanreks (Echinops telfairi Martin). Zeitschrf. f. Parasitenkd, XXII (2), 176-182 (fig. 1-5).

Johnston (T. H.) et Edmonds (S. J.), 1952. - Australian Acanthocephala, n 9, Transac. Roy. Soc. South Australia, LXXV, 16-21 (fig. 1-17).

LiNstow (Otton von), 1898. - Nemathelminthen von Herrn Richard Semon in Australien gesammelt. Denkr. Med. Nat. Gesch. Jena, VIII, 467-472 (pl. XXXV, fig. n 16 à 29).

- - 1897. - Nemathelminthen grösstentheils in Madagascar gesammelt. Arch. f. Naturgesch, LXIII, Jahg, Bd 1 (Heft 1), 27-34 (pl. 4 et 5, fig. 1-26).

- - 1902. - Beobachtungen an neuen und bekannten Nemathelminthen. Arch. iv. Mikrobiolog. Anatom. Jahrg., LX, 217-232 (+ pl. XIII, fig. 17 à 34).

MeYer (Anton), 1932. - Acanthocephala. Dr H. G. Bronn's Klassen und Ordnungen des Tierreichs, IV, Bd, 2 Abt. 2 Buch Leipzig, pp. 1-332 (fig. 1-306).

Müller (Otto Frd), 1776. - Zoologicae Danicae seu Animalium Daniae et Norvegia Indigenarum Characteres, Nomina et Synonyma imprimis popularium, $8^{\circ}$, Havniae, 214-215.

Petrotschenko (V. I.), 1958. - Acanthocéphales des animaux sauvages et domestiques (en russe). Tome II. Akad. Nauk. S.S.S.R., 458 pp. (fig. 1-178).

Travassos (Lauro), 1915. - Revisaro dos Acantocefalos brazileiros. I. Fam. Gigantorhynchidae, Hamann 1892 (2 a nota previa). Brazil Medic. Rio-de-Janeiro, XXIX, $\mathrm{n}^{\circ *} 48$, 18 , 12 , p. 377.

Van Cleave (Harley J.), 1924. - A critical Study of the Acanthocephala described and identified by Joseph Leidy. Proceed. Acad. Nat. Sci. Philadelphia, LXXVI, 279-334 (pl. 14-18).

WARD (Henry B.), 1917. - Echinorhynchus moniliformis in North America. Jl. Parasitol., III (3), 141.

Travail de l'Institut de Parasitologie comparée de l'Ecole pratique des Hautes Etudes

(Directeur émérite : Robert $\mathrm{Ph}$. Dollfus)

et de l'Institut de Parasitologie de la Faculté de Médecine de Paris

(Directeur : Lucien Ch. BRUMPT) 